\title{
Quantentheoretische Bemerkungen zum Experiment von Stern und Gerlach.
}

\author{
Von A. Einstein in Berlin und P. Ehrenfest in Leiden.
}

(Eingegangen am 21. August 1922.)

§ 1. O. Stern und W. Gerlach ${ }^{1}$ ) ließen einen Dampfstrabl von Silberatomen durch ein Magnetfeld fliegen um festzustellen, ob die Atome ein magnetisches Moment besitzen und - wenn ja - welche Orientierung dasselbe während der Durchquerung des Magnetfeldes aufweist. Ihr Experiment liefert als sehr bedeutsames Resultat: das magnetische Moment aller Atome fällt während der Durchquerung des Feldes mit der Richtung der Kraftlinien zusammen, und zwar für etwa die Hälfte der Atome im Sinne des Feldes, für die andere Hälfte entgegengesetzt. Es drängt sich natürlich die Frage anf, auf welche Weise die Atome zu dieser Orientierung gelangen.

§ 2. Dabei ist vor allem zu beobachten, daß die Atome während ihres Eintretens in das ablenkende Magnetfeld keine Zusammenstöße erfahren - die letzten Zusammenstöße erfahren sie ja im Dampfraum des Schmelzöfchens.

Wir fragen uns zunächst, wie magnetische Atome unter dem Einfluß eines magnetischen Feldes überhaupt ihre Orientierung ändern. Solange man von Strahlungsemission und -absorption, Zusammenstößen oder anderen ähnlichen Einflüssen absieht, führen die Atome im Magnetfeld eine Präzessionsbewegung (Larmor-Rotation) um die Richtung des Feldes aus. Ändert sich die Richtung des Feldes langsam gegenüber der Raschheit der Präzessionsbewegung, so bleibt der Winkel der Präzessionsbewegung anverändert bestehen. Eine Einstellung in die durch die Quantentheorie geforderten Neigungen $(O$ und $\pi$ für das Silberatom nach dem Experiment von Stern und Gerlach) kann demnach ohne äußere Einflüsse von der Art der Strahlung oder der Zusammenstöße nicht stattfinden.

§ 3. Die nächstliegende Erklärung für den experimentellen Befund scheint im ersten Augenblick die za sein, daß die Einstellung der Atome beim Eintritt in das Feld des Elektromagnetes erfolgt, und zwar durch Strahlungsaustausch. Es ist dann nicht nur Energieabgabe, sondern auch Energieaufnahme ans dem Strahlungsfeld erforderlich, nämlich letztere für die Atome, die sich antiparallel zu den Kraftlinien stellen. Wie rasch erfolgt nun die Umlegung der Atom-

1) ZS. f. Phys. 9, 349, 1922. 
momente unter dem Einfluß der Strahlung (von Zimmertemperatur)? Verhältnismäßig sicher läßt sich die erforderliche Zeit abschätzen für den Fall von Übergängen von Quanten- nach Quantenzuständen. Denn wir wissen, daß in Fällen dieser Art für den Utbergang einer Schaar von Atomen diese Zeit jeweils - wenigstens der Größenordnung nach - übereinstimmt mit der eines entsprechenden klassischen Modells. In unserem Fall eines präzessionierenden Atoms mit magnetischem Moment wäre das ein bei seiner konischen Rotation ausstrahlender magnetischer Dipol. Die Einstellungszeit würde (bei einer Feldstärke von $10000 \mathrm{Gauß)}$ von der Ordnung von $10^{11}$ Sek. sein, falls allein die Ausstrahlung der Präzessionsbewegung wirksam wäre. Beachtet man aber den Einfluß der umgebenden Zimmertemperaturstrahlung [„positive und negative Einstrahlung(1)], so verkürzt sie sich auf etwa $10^{9}$ Sek.

Das sind also jedenfalls Zeiten von einer Größenordnung, die für das Experiment überhaupt nicht in Betracht kommen, denn hier muß die Einstellung in einer Zeit von weniger als $10^{-4}$ Sek. erfolgen.

$\S 4$. Wenn man versucht aus dieser Schwierigkeit herauszukommen, so bieten sich zunächst zwei alternative Annahmen dar:

A. Der wirkliche Mechanismus ist derart, daß Atome niemals in einen Zustand geraten können, in welchem sie nicht vollständig quantisiert sind.

B. Bei raschen Einwirkungen resultieren Zustände, die bezüglich der Orientierung die Quantenregel verletzen; die von der Quantenregel verlangten Einstellungen werden durch Aus- and Einstrahlung hergestellt, und zwar mit einer Reaktionsgeschwindigkeit außerordentlich viel größer als bei Utbergängen von Quanten- nach Quantenzuständen.

Eine Entscheidung a priori zwischen diesen beiden Altermativen scheint gegenwärtig nicht möglich zu sein; aber es ist angezeigt sich ihren prinzipiellen Unterschied und die charakteristischen Schwierigkeiten, zu denen jede von ihnen führt, deutlich vor Augen zu bringen.

$\S 5$. Besprechung der Alternative A. 1. Was sie fordert, läßt sich besonders gut am Experiment von Stern-Gerlach exemplifizieren: Im Dampfraum des Schmelzöfchens ist jedes Silberatom: unmittelbar nach jedem Zusammenstoß vollständig quantisiert, also seine magnetische Achse nach dom dort herrschenden, wenn auch noeh so schwachen magnetischen Feld orientiert. Nach seinem letzten

1) Vergl. A. Einstein, Zur Quantentheorie der Strahlung. Phys. ZS. 18, 121, 1917, §2. 
Zusammenstoß während seines Fluges durch die verschiedenen Teile des Feldes bleibt seine Orientierung fortwährend angepaßt an die Feldrichting des betreffenden Ortes ${ }^{1}$ ).

2. Dabei wird ein Teil der (einquantigen) Momente parallel, ein anderer antiparallel mit dem Feld stehen, wobei die statistische Verteilung durch die Temperatur und Feldstärke im Dampfraum des Öfchens beherrscht wird und durchaus nicht durch die (Strahlungs-) Temperatur und die Feldstärken im weiterhin durchflogenen Raum!

3. Man müßte sich demnach entschließen folgendes anzunehmen: Noch so schwache Felder müssen unmittelbar nach dem Zusammenstob (d. h. der Wirkung sehr starker Felder) für die Orientierung entscheidend sein. Bei Änderungen, z. B. der Feldrichtung des magnetischen Feldes, welche beliebig rasch sind gegenüber der Larmorrotation, sollte also die magnetische Achse des Atoms der Feldrichtung ebenso vollkommen folgen, als bei beliebig langsamen Änderungen. Allgemeiner: Bei beliebig rascher Veränderung der äußeren Bedingungen eines mechanischen Systems müßte sich dieses in demselben Endzustand einstellen, wie bei unendlich langsamer (adiabatischer) Durchführung der Veränderung der äußeren Bedingungen. Daß dieses eine Verletzang der mechanischen Gleichungeu verlangt, kann man sich leicht an Beispielen deutlich machen ${ }^{2}$ ).

\$ 6. Besprechung der Alternative B. 1. Für das Experiment von Stern-Gerlach würde sich folgendes Bild ergeben: Im Dampfraum des Ofchens ist unmittelbar nach jedem Zusanmenstoß die magnetische Achse eines Atoms willkürlich bezüglich des dort anwesenden schwachen Feldes orientiert. Die Orientierung erfolgt durch ultrarote Strahlung, and zwar durch Ausstrahlung und positive und negative Einstrahlung mit einer Einstellung parallel und antiparallel zum Feld. Dabei ist die Voranssetzung wesentlich, daß derartigen Übergängen von Nichtquanten- in Quantenzustände Umsetzungswabr-

1) Dr. G. Breit schlug schon gelegentlich einer Diskussion im physikalischen Kolloquium in Leiden eine derartige Annahme vor.

2) Ein einigermaßen fiktives Beispiel: Eine adiabatische Verkürzung der Fadenlänge eines Schwerependels verändert bekanntlich die Frequenz $\boldsymbol{\nu}$ und die Energie $\varepsilon$ konform so, daß die Quantenregel erfüllt bleibt. Verkürzt man hingegen rasch, z. B. bei vertikaler Stellung, die Fadenlänge, so wird $\boldsymbol{y}$ größer, während nach der Mechanik Energie nicht zugeführt wird. Die Alternative A verlangt also eine mechanisch unbegreifliche Arbeitszufuhr. - Zweites Beispiel: Ein magnetisches Atom in schwachem Magnetfeld. Bei einer unendlich langsamen Drehung des Feldes (unendlich langsam gegen Präzessionsgeschwindigkeit) folgt nach den Gesetzen der Mechanik die magnetische Achse des Atoms der Feldrichtung. Soll dies ebenso bei rascher Änderung der Feldrichtung geschehen, so liegt eine mechanisch unbegreifliche Änderung des Drehimpulses vor.

Zeitschrift für Physik. Bd. XI. 
scheinlichkeiten entsprechen, von einer viel höheren Größenordnung ${ }^{1)}$ als bei Obergängen von Quanten- zu Quantenzuständen. Nach dem letzten Zusammenstoß paßt sich beim Fluge durch die verschiedenen Teile des Feldes die Orientiernng der Achse quasiadiabatisch an die wechselnde Feldrichtung an, in dem die jeweils eingetretenen sehr kleinen Winkeldefekte mit Hilfe eines äußerst schwachen Strahlnngsaustausches von sehr ultraroter Frequenz (noch viel mehr ultrarot als die Präzessionsfrequenz) ansgeglichen werden.

2. Die statistische Verteilung zwischen Orientierung parallel und antiparallel zum Feld würde auch in diesem Fall im wesentlichen durch Temperatur und Feldstärke im Schmelzöfehen bestimmt sein!

3. Nach der Alternative B würde ein einatomiger Dampf, dessen Atome magnetische Momente tragen, im Magnetfeld emittieren und absorbieren atf der langwelligen Seite der Frequenz der Präzessionsbewegnng; also bei passendem Magnetfeld im Gebiete elektrischer Wellen.

4. Charakteristisch für die Alternative $B$ ist, daß sie die Anpassung an Quantenzustände abhängig macht von der Möglichkeit einer Ein- und Ausstrahlung. Sie macht also einen prinzipiellen Unterschied zwischen rein mechanischen und strahlungsfähigen Systemen. Z. B. sollte die Drehachse eines symmetrischen schweren Kreisels nur dann bezüglich des Schwerefeldes Quanteneinstellung erreichen können, wenn der Kreisel passende elektrische Ladungen trägt. Wollte man vollends die Hypothese B von der Einstellung bezüglich Orientierung auf Einstellung in Quantenzustände überhaupt ausdehnen, d. h. also z. B. auch den Schwingungen eines Kristallgitters und den Rotationen eines Moleküls eine spontane Einstellung auf Quantenbahnen nur im Falle passender elektrischer Ladungen erlauben, so käme man in evidenten Widerspruch mit den Erfahrungen bezüglich der spezifischen Wärmen, z. B. von Diamant und gasförmigem $\mathbf{H}_{2}$.

\& 7. Die aufgezählten Schwierigkeiten zeigen, wie unbefriedigend die beiden hier besprochenen Deutungsversuche der von Stern und Gerlach gefundenen Resultate sind. Bohrs Auffassung - daß in komplizierteren Feldern überbaupt keine scharfe Quantisierung besteht - blieb unbesprochen.

Leiden-Berlin, Mai-Juni 1922.

1) Entsprechend einer Einstellungszeit von $10^{-4}$ statt $10^{9}$ Sek. 